\title{
Non-Newtonian viscosity of interacting Brownian particles: comparison of theory and data
}

\author{
Matthias Fuchs $^{1,3}$ and Michael E Cates ${ }^{2}$ \\ ${ }^{1}$ Institut Charles Sadron, 6, rue Boussingault, 67083 Strasbourg Cedex, France \\ ${ }^{2}$ Department of Physics and Astronomy, The University of Edinburgh, JCMB King's Buildings, \\ Edinburgh EH9 3JZ, UK
}

Received 16 October 2002

Published 16 December 2002

Online at stacks.iop.org/JPhysCM/15/S401

\begin{abstract}
A recently developed first-principles approach to the non-linear rheology of dense colloidal suspensions is evaluated and its results compared to those from simulations of sheared systems close to their glass transitions. The predicted scenario of a universal transition of the structural dynamics between yielding of glasses and non-Newtonian (shear-thinning) fluid flow appears well obeyed, and calculations within simplified models rationalize the data over variations in shear rate and viscosity of up to three decades.
\end{abstract}

(Some figures in this article are in colour only in the electronic version)

\section{Introduction}

The rheological properties of soft materials, such as colloidal dispersions, presumably originate from a number of physical mechanisms, such as shear-induced phase transitions, direct potential and hydrodynamic interactions, advection of fluctuations, and shear banding or localization; see e.g. the collection of papers in [1]. At higher particle concentrations, the non-linear rheology depends on how steady shearing interferes with solidification during glass formation. Recently, we developed a theory for the non-linear rheology of dense colloidal suspensions aimed at addressing this point [2]. It describes how the structural dynamics is fluidized by advection of density fluctuations, while hydrodynamic interactions, non-linear flow profiles, and ordering phenomena are neglected. Computer simulation studies can ensure that the latter processes are absent and thus provide crucial tests of the scenario presented. In this contribution, theoretical calculations will be compared to Brownian dynamics simulations of hard spheres by Strating [3] — without an adjustable parameter in principleand to molecular dynamics simulations of a sheared binary Lennard-Jones mixture by Berthier and Barrat [4].

3 Permanent address: Physik-Department, Technische Universität München, James-Franck-Str., 85747 Garching, Germany. 


\section{Theory}

\subsection{General aspects}

A system of Brownian particles is studied in a prescribed steady-shear solvent flow with constant velocity gradient and shear rate $\dot{\gamma}$. The equation of motion for the temporal evolution of the many-particle distribution function is known [5], and has been solved for hard spherical particles at low densities [6]. This model constitutes a first microscopic approach to real dense colloidal suspensions, and may serve as a model sheared glassy fluid [7]. It considers the 'Brownian part' of the viscosity only, which, in Stokesian dynamics simulations, Foss and Brady [8] found to dominate compared to the hydrodynamic one for small shear rates.

While the (approximate) approach developed in [2] gives general steady-state quantities (such as the shear-distorted static structure factor) and their time-dependent fluctuations close to glassy arrest, we will concentrate on the thermodynamic shear stress $\sigma(\dot{\gamma})$ and the connected shear viscosity $\eta(\dot{\gamma})=\sigma / \dot{\gamma}+\eta_{\infty}$; here $\eta_{\infty}$ is the viscosity of the background solvent. The equations of motion exhibit a glass transition bifurcation, around which asymptotic expansions capture the transition from shear-thinning fluid flow to solid-like yielding. With the separation parameter $\varepsilon$ denoting the (relative) distance from the transition, and $t_{0}$ a timescale obtained by matching to microscopic transient motion, the following behaviours of $\sigma$ in the "structural window' have been established [2]:

$$
\sigma=\sigma\left(\dot{\gamma} t_{0}, \varepsilon\right) \rightarrow \begin{cases}\dot{\gamma} t_{0}|\varepsilon|^{-\gamma} c_{1} & \varepsilon<0 \\ c_{2}\left(1+c_{3}\left|\dot{\gamma} t_{0}\right|^{m}\right) & |\varepsilon| \ll\left|\dot{\gamma} t_{0}\right|^{\frac{2 a}{1+a}} \\ c_{2}\left(1+c_{4} \sqrt{\varepsilon}\right) & \varepsilon>\left|\dot{\gamma} t_{0}\right|^{\frac{2 a}{1+a}},\end{cases}
$$

where the $c_{i}$ are positive material-dependent parameters (for the exponents $\gamma$ and $a$, see e.g. [9]). The 'structural window', here, is defined as the double regime $|\varepsilon| \ll 1$ and $\left|\dot{\gamma} t_{0}\right| \ll 1$, where the slowing down of the structural dynamics dominates the steady-state stress. While the divergence of the Newtonian viscosity $\eta_{0}=t_{0}|\varepsilon|^{-\gamma} c_{1}$ (first line of equation (1)), upon approaching the transition, applies to the linear response regime of a fluid $(\varepsilon<0)$, and is known from mode-coupling theory (see the references in [9]), the novel predictions close to and above $(\varepsilon \geqslant 0)$ the transition describe the universal non-linear response of glasses to steady shearing with rate $\dot{\gamma}$. Importantly, a 'dynamic yield stress' $\sigma^{+}(\varepsilon)=\sigma(\dot{\gamma} \rightarrow 0+, \varepsilon \geqslant 0)$ is obtained, because a finite stress has to be overcome in order to force the glass to yield even for vanishingly small shear rate; $\sigma^{+}$is connected to the constants $c_{2}$ and $c_{4}$ in equation (1). While the yield stress varies strongly with distance to the transition deep in the glass, at fixed parameters close to the transition, the stress increases from $\sigma^{+}$with a power law in $\dot{\gamma}$, where the material-dependent exponent $m$ lies around 0.15 in the models studied below. The given asymptotes are only the leading orders for $\varepsilon \rightarrow 0$ and $\dot{\gamma} t_{0} \rightarrow 0$, while corrections can be obtained systematically [2], or are included in model calculations to be presented below.

The dominance of the structural dynamics in determining $\sigma\left(\dot{\gamma} t_{0}, \varepsilon\right)$ entails that all exponents or constants are functions of the equilibrium structure factor $S_{q}$ alone, except for the timescale $t_{0}$ which matches to shorter non-structural dynamics. Thus, hydrodynamic interactions or inertial terms only influence the value of $t_{0}$, which ideally could be determined from an analysis of the intermediate-scattering functions of the system [9]. This result arises because the small-shear-rate rheology of glassy suspensions is dominated by steric hindrance (the 'cage effect') which is not qualitatively affected by the properties of the solvent around the particles. This is in agreement with the finding in Stokesian dynamics simulations [8] that shear thinning is dominated by a decrease of the Brownian part of the viscosity. The elimination of particle forces in favour of the quiescent-state structure factor $S_{q}$ is an approximation of unknown quality in the present situation, but is in part motivated by the consideration of small shear rates. 


\subsection{Models and simplifications}

The equations of motion, from which $\sigma$ in equation (1) follows uniquely for a given $S_{q}$, have not been solved yet. Two approximate models were presented and discussed in [2] and will be used in the following. While the schematic $F_{12}^{(\dot{\gamma})}$-model only incorporates the competition of two effects (divergent structural relaxation times with increasing $\varepsilon$ and loss of memory induced by shearing), the semi-microscopic ISHSM combines a semi-quantitative description of a quiescent hard-sphere colloidal dispersion [9] with an isotropically averaged shear advection of density fluctuations. Both models depend on only two parameters which map onto $\varepsilon$ and $\dot{\gamma}$ introduced in equation (1), and thus can be viewed as minimal models for the scenario described.

A problem when analysing data using either model arises from the ratio $c_{2} / c_{1}$ in equation (1), which has a simple physical meaning. It gives the ratio of yield stress to transverse elastic constant (namely the shear modulus $G_{\infty}$ ) of the glass at the critical point, $c_{2} / c_{1}=\hat{c}_{1} \sigma_{c}^{+} / G_{\infty}^{c}$, where the numerical constant $\hat{c}_{1}=1.0(1)$ for the $\operatorname{ISHSM}\left(F_{12}^{(\dot{\gamma})}\right)$. This ratio can be interpreted as a critical yield strain. Both models underestimate the effect of shearing leading to $\sigma_{c}^{+} / G_{\infty}^{c}=0.33(0.34)$ for the $\operatorname{ISHSM}\left(F_{12}^{(\dot{\gamma})}\right)$, while experiments give values around 0.05 indicating that smaller strains are necessary for yielding [10]. While the schematic $F_{12}^{(\dot{\gamma})}-$ model is not meant to quantitatively capture such ratios, this error in the ISHSM presumably arises from the oversimplified handling of the shear-induced anisotropy of density fluctuations. The ISHSM treats all directions equivalent to the vorticity direction that is perpendicular to the flow plane. Perhaps unsurprisingly, this underestimates the effects of shearing. We correct for this error in an ad hoc fashion by rescaling the shear rate $\dot{\gamma}$ when considering $\eta(\dot{\gamma})$. For the $F_{12}^{(\dot{\gamma})}$-model this procedure is rigorously equivalent to an adjustment of the ratio $\sigma_{c}^{+} / G_{\infty}^{c}$.

\section{Results and comparison with simulation data}

Before applying equation (1) to a solution of colloidal hard spheres at packing fraction $\phi$, at first the latter's critical value, $\phi_{c}$, entering in $\varepsilon=C\left(\phi_{c}-\phi\right) / \phi_{c}$ (with $C=1.5$ [9]), needs to be determined. This is done by testing whether the divergence of the quiescent viscosity (and corresponding structural relaxation time) for $\varepsilon \rightarrow 0-$ is observed. The inset of figure 1 shows viscosities from experiments [11] and from Brownian dynamics simulations [3]. Also included are self-diffusion coefficients from [12], which are predicted to vanish with $D \propto|\varepsilon|^{\gamma}$. Replotting the data with the calculated $\gamma=2.62$ [9], fits to the data above $\phi \geqslant 0.50$ give $\phi_{c}=0.57$ [12], 0.58, and 0.60 for $D, \eta$ (two outliers neglected), and simulations, respectively. Interestingly, the two experimental data sets provide rather close estimates for $\phi_{c}$ and indicate a strong coupling of diffusion and viscosity, $D \eta \rightarrow 0.4 \eta_{\infty} D_{0}$ for $\varepsilon \rightarrow 0$ (neglecting the difference in $\phi_{c}$ ), with $D_{0}$ the dilute single-particle diffusion coefficient. The numerical factor is about half the predicted value [9]. We speculate that the discrepancy of the extrapolation of the simulation results arises in part because the data are not fully in the asymptotic regime.

With the quantitative knowledge of $\varepsilon$, only the matching time $t_{0}$ is required to analyse the steady-state viscosities in the structural window using the ISHSM. We chose to obtain it via the full fitting procedure which consists in matching by eye the numerical solutions to the non-Newtonian viscosity data. In this way, $t_{0}$ is mainly determined by the increase of the Newtonian viscosity, because $\eta \sim t_{0} \sigma /\left(\dot{\gamma} t_{0}\right)$ holds and $\sigma /\left(\dot{\gamma} t_{0}\right)$ becomes independent of $t_{0}$ in the fluid for vanishing shear rate. The main panel of figure 1 shows $\eta$ from the Brownian dynamics simulations as function of the dimensionless Peclet number $P e_{0}=\dot{\gamma} d^{2} / D_{0}$, which measures the effect of shearing relative to the time taken for a single particle to diffuse over its 


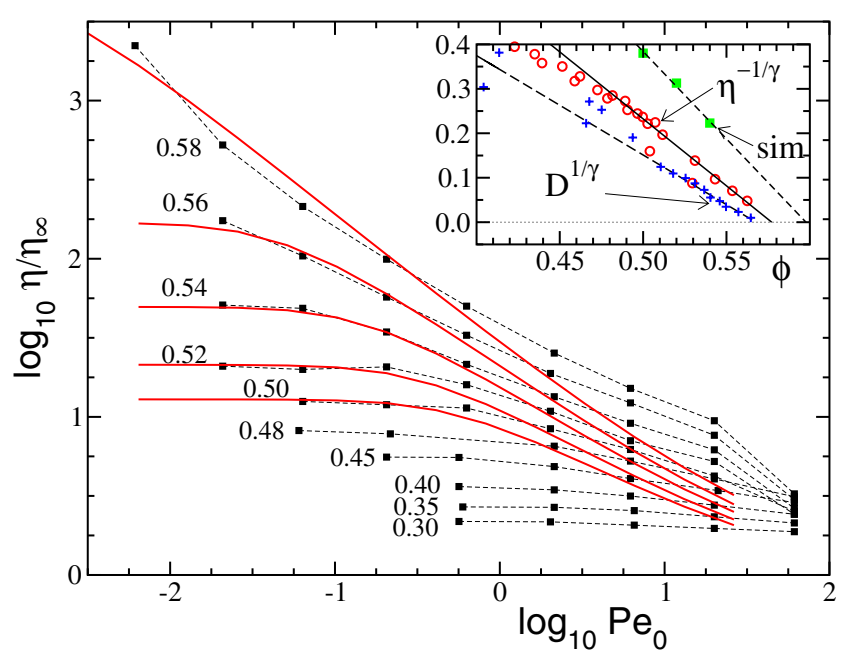

Figure 1. Steady-state viscosities (symbols) from Brownian dynamics simulations [3] versus Peclet number $P e_{0}=\dot{\gamma} d^{2} / D_{0}$ for packing fractions $\phi$ as labelled. Fits by eye to the data for $\phi \geqslant 0.50$ with the ISHSM for separation parameters $-\varepsilon=0.014,0.058,0.097,0.139$, and 0.174 are given as solid lines and extrapolate to $\phi_{c}=0.59$. The matching time $t_{0}=0.019 d^{3} \eta_{\infty} / k_{B} T$ is obtained and the theoretical $\dot{\gamma}$ is rescaled to $0.25 \dot{\gamma}$ as discussed in the text. The inset shows a rectification plot with predetermined exponent $\gamma=2.62$ of viscosities from experiments [11] (circles) and simulations [3] (squares), alongside self-diffusion constants (crosses) from [12], versus packing fraction. Linear fits to the data above $\phi \geqslant 0.50$ give $\left(\eta / \eta_{\infty}\right)^{-1 / \gamma}=1.2 \varepsilon$ with $\phi_{c}=0.58$ (two outliers neglected), $\left(\eta / \eta_{\infty}\right)^{-1 / \gamma}=1.6 \varepsilon$ with $\phi_{c}=0.60$, and $\left(D_{L} / D_{0}\right)^{1 / \gamma}=0.8 \varepsilon$ with $\phi_{c}=0.57$.

diameter $d$. The fits by eye using the ISHSM are included for packing fractions close to the transition, $\phi \geqslant 0.50$. From the fits, mainly from the divergence of $\eta_{0}$ given in equation (1), the matching time $t_{0}=0.019 d^{3} \eta_{\infty} / k_{B} T$ is estimated, and inclusion of corrections to asymptotic behaviour in the ISHSM fits shifts the glass transition packing fraction closer to the other determinations; $\phi_{c}=0.59$ follows from the $\varepsilon$ used in figure 1 . Note that the solvent viscosity is included in the theoretical curves ${ }^{4}, \eta=\eta_{\infty}+t_{0} \sigma /\left(\dot{\gamma} t_{0}\right)$. In the shear-thinning region, the viscosity diminishes and approaches a behaviour like $\eta \sim \sigma_{c}^{+} / \dot{\gamma}$, with strong corrections, though, masking the power law [2]. Because of the overestimate of $\sigma_{c}^{+}$in the ISHSM, this decrease would set in at too high $\dot{\gamma}$-values only. In order to correct for the quantitative error, the theoretical curves are plotted versus rescaled shear rate, $\dot{\gamma} * 0.25$; i.e. $\sigma /\left(\dot{\gamma} t_{0}\right)=f_{\eta}(\dot{\gamma} * 0.25)$. With this ad hoc correction, satisfactory agreement of theory and simulation results is seen for $P e_{0} \leqslant 1$, where the steady-state viscosity varies over two orders on variation of the shear rate and packing fraction. For larger Peclet numbers, the data presumably lie outside the structural window where equation (1) applies. Motivated by numerical findings in [2], we speculate that the enhanced, $\phi$-dependent steady-state viscosities around $P e_{0}=1-10$ in the Brownian dynamics simulations originate from the hard-core repulsion. If so, hydrodynamic interactions which prevent particles from close contact could appreciably affect $\eta$ in this region.

A second set of steady-state shear stresses and viscosities is provided by recent large-scale molecular dynamics simulations of a sheared simple liquid (a binary Lennard-Jones mixture) above and below its glass transition temperature [4]. Because kinetic parameters do not enter

4 The ISHSM calculations provide $\sigma\left(\varepsilon, \dot{\gamma} t_{0}, P e_{0}\right)$ for all values of $\varepsilon$ and $P e_{0}$, while equation (1) captures the asymptotic behaviour for $\varepsilon \rightarrow 0, \dot{\gamma} t_{0} \rightarrow 0$, and $P e_{0} \rightarrow 0$. Because we aim at describing the proximity of the glass transition, we match the parameters of equation (1) $\left(\phi_{c}\right.$ and $\left.t_{0}\right)$ there. Without matching, the ISHSM gives $\phi_{c}=0.52$ and $t_{0}=0.025 d^{3} \eta_{\infty} / k_{B} T$. 


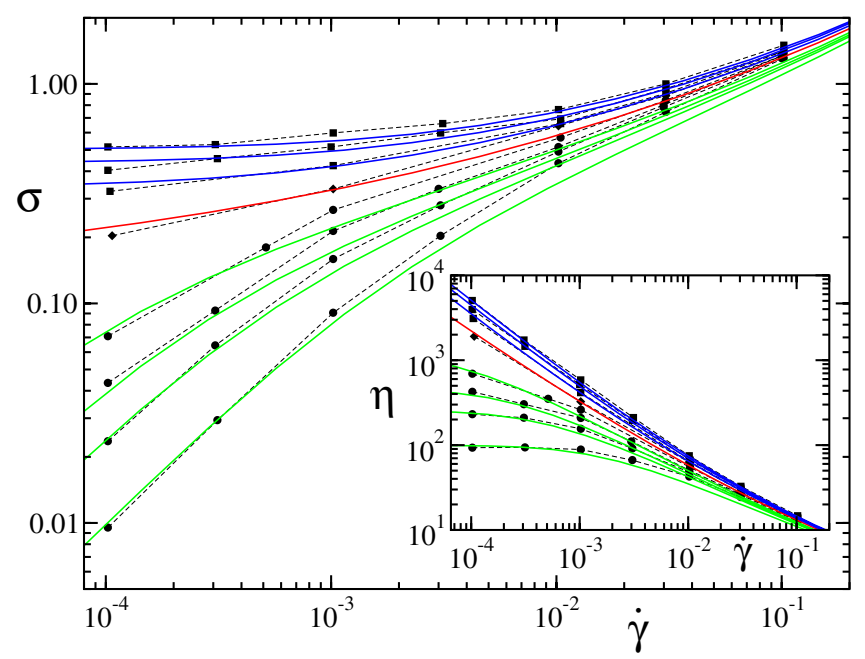

Figure 2. Symbols represent shear stress (main panel) and viscosity (inset) data for a supercooled Lennard-Jones binary mixture in reduced units taken from [4]; from top to bottom, the temperatures are $0.15,0.3,0.4,0.45,0.5,0.525,0.555$, and 0.6 while $T_{c} \approx 0.435$. The solid curves give fits obtained by eye using the $F_{12}^{(\dot{\gamma})}$-model from [2] with separation parameters: $\varepsilon=0.050,0.037$, $0.021,0.0,-0.027,-0.042,-0.054$, and -0.083 (from top to bottom); the units are converted using $\sigma=\dot{\gamma} \eta=1.8\langle\tau\rangle \dot{\gamma}$, where $\dot{\gamma}=0.53 P e_{0}$, and $\langle\tau\rangle=\left\langle\tau\left(\varepsilon, P e_{0}\right)\right\rangle[2]$

the theoretical predictions, and as linear flow profiles were obtained in the simulations, the universal predictions of our approach can be compared again. Figure 2 shows stationary shear stresses from the simulations and fits by eye using the $F_{12}^{(\dot{\gamma})}$-model as specified in [2]. The model provides a relaxation time $\langle\tau\rangle$ as a function of $\varepsilon$ and a dimensionless shear rate, denoted as the Peclet number $P e_{0}$, which are mapped onto the data as specified in the figure caption. The data nicely span the glass transition temperature, $T_{c} \approx 0.435$, already known [4], and agree well with a transition from a shear-thinning fluid to a yielding glass with finite yield stresses at and below the transition temperature.

\section{Conclusions and outlook}

We presented results of a microscopic theory of the non-linear rheology of colloidal fluids and glasses under steady shear [2], and compared them with simulation and experimental data. This brought out the existence of a universal transition between shear-thinning fluid flow, with diverging viscosity upon increasing the interactions, and solid yielding, with a yield stress that is finite at (and beyond) the glass point. Numerical calculations were able to explain simulation results over up to three decades of variation in the shear rate and viscosity. A quantitative analysis of larger data sets is required in order to determine the theoretical parameters for both simulations more accurately than the estimates found here.

The approach that we outlined should be improved as regards the handling of shear-induced anisotropies, and stress-induced effects. The latter may lead to shear-thickening behaviour that, for many colloidal materials, occurs at higher flow rates than those addressed here. This avenue will be explored in a future paper [13] on a version of the schematic model which is modified to include explicit stress rate (as well as strain rate) dependence. 


\section{Acknowledgments}

We thank J-L Barrat and L Berthier for discussions. MF was supported by the DFG, grant Fu 309/3.

\section{References}

[1] Pusey P N (ed) 2002 Faraday Discuss. 123

[2] Fuchs M and Cates M E 2002 Phys. Rev. Lett. at press

(Fuchs M and Cates M E 2002 Preprint cond-mat/0204628)

Fuchs M and Cates M E 2002 Faraday Discuss. 123 at press

(Fuchs M and Cates M E 2002 Preprint cond-mat/0207530)

[3] Strating P 1999 Phys. Rev. E 592175

[4] Berthier L and Barrat J L 2002 J. Chem. Phys. 1166228

[5] Dhont J K G 1996 An Introduction to Dynamics of Colloids (Amsterdam: Elsevier)

[6] Bergenholtz J, Brady J F and Vicic M 2002 J. Fluid Mech. 456239

[7] Fielding S M, Sollich P and Cates M E 2000 J. Rheol. 44323

[8] Foss D and Brady J F 2000 J. Fluid Mech. 407167

[9] Franosch T, Fuchs M, Götze W, Mayr M R and Singh A P 1997 Phys. Rev. E 557153 and references therein

[10] Russel W B, Saville D A and Schowalter W R 1989 Colloidal Dispersions (New York: Cambridge University Press)

[11] Cheng Z, Zhu J, Chaikin P M, Phan S-E and Russel W B 2002 Phys. Rev. E 65041405

[12] van Megen W, Mortensen T C, Williams S R and Müller J 1998 Phys. Rev. E 586073

[13] Holmes C B, Fuchs M and Cates M E 2002 in preparation 